\title{
XCV. The reflexion of light at an ideal plane mirror moving with a uniform velocity of translation
}

\section{H. Bateman}

To cite this article: H. Bateman (1909) XCV. The reflexion of light at an ideal plane mirror moving with a uniform velocity of translation, Philosophical Magazine Series 6, 18:108, 890-895, DOI: 10.1080/14786441208636765

To link to this article: http://dx.doi.org/10.1080/14786441208636765

曲 Published online: 21 Apr 2009.

Submit your article to this journal $₫$

Џ Article views: 9

Q View related articles $\square$ 
XCV. The Reflexion of Lught at an Ideal Plane Mirror moving uith a Uniform Velocity of Translation. By $\mathrm{H}$. BATEMAN *.

TTHE question whether the motion of a mirror has any effect on the laws of reflexion and refraction was considered by Fresnel $\dagger$, who came to the conclusion that no discrepancy need be expected. Stokes $f$, examining the question in a more general manner, showed that the laws of reflexion and refraction are satisfied provided the directions of the rays are measured relative to the mirror and quantities of an order bigher than the first, in the ratio of the velocity of the mirror to that of light, are neglected.

The question was brought into prominence in the discussions of the Michelson-Morley experiment, and is of fundamental importance in the theory of radiation $\S$, where we have to consider the case in which the mirror is moving relatively to the observer.

An exact theory of reflexion is required for a rigorous discussion of the Michelson-Morley experiment, Wien's law, the pressure of radiation on a moving mirror, and the effect of motion on the temperature of radiation. The precise laws of reflexion have accordingly been discussed by Voigt $\|$, Lodge $\uparrow$, Hicks **, Abraham $\dagger \dagger$, Einstein $\ddagger \ddagger$, Planck $\$ \S$, von Mosengeil || |, and Kohl $\mid$ I.

The object of this paper is to make a small addition to the results obtained by these writers. It will be convenient to use them in the form obtained by Hicks, Abrahan, and Einstein.

Let $c$ be the velocity of light in free æther, $w$ the velocity of the mirror referred to a set of axes which are at rest relatively to the observer. Let $v$ be the component of $w$ along

* Communicated by the Author.

$\dagger$ Annales de Chimie, t. ix. (1818) p. 57.

$\ddagger$ Phil. Mag. vol. xxvii. (1846) p. 76.

$\$$ Larmor, British Association Reports, 1900, p. 657. Encyclopadia Britannica, article 'Radiation,' in vol. xxxii. (1903). Boltzmann, Festschrift, p. 590 (1904).

II Götinger Nachrichten, 1887, p. 215.

T Phil. Trans. A. (1893) p. 793.

** Phil. Mag. [6] iii. (1902) p. 9.

t† Boltzmann, Festschrift, p. 85 (1904); Ann. d. Phys. [4] xiv. p. 236 (1904): Theorie der Elektricität, vol. ii. p. 343.

It Ann. d. Phys. [4] xvii. (1905).

$\$ \$$ Theorie der Wïrmestrahlung, p. 71.

Ann. d. Phys. [4] xxii. (1907).

II Ibid. xxviii. p. 28 (1909). 
the normal to the mirror, the positive direction of the norma? being on the reflecting side of the mirror.

Take the axis of $x$ along the normal and let the electric rector in the incident wave be given by

$$
\mathrm{E}=e \sin v\left[t+\frac{x \cos \phi+y \sin \phi}{c}\right],
$$

where $e$ is independent of $(x, y, z, t)$.

This represents a plane wave of light of frequency $*$ travelling in a direction opposite to that in which the mirror is moving. The normal to the wave front makes an angle $\pi+\phi$ with the normal to the mirror, so that the angle of incidence is $\phi$.

The reflected wave is given by

where *

$$
\mathrm{E}^{\prime}=e^{\prime} \sin \nu^{\prime}\left[t-\frac{x \cos \phi^{\prime}-y \sin \phi^{\prime}}{c}\right],
$$

so that

$$
\left.\begin{array}{rl}
\nu \cos \phi & =v^{\prime}\left[-\frac{2 c v}{c^{2}-v^{2}}+\frac{c^{2}+v^{2}}{c^{2}-v^{2}} \cos \phi^{\prime}\right] \\
v \sin \phi & =v^{\prime} \sin \phi^{\prime} \\
\nu & =v^{\prime}\left[-\frac{2 c v}{c^{2}-v^{2}} \cos \phi^{\prime}+\frac{c^{2}+v^{2}}{c^{2}-v^{2}}\right]
\end{array}\right\}
$$

$$
\begin{gathered}
\cos \phi=\frac{\left(c^{2}+v^{2}\right) \cos \phi^{\prime}-2 c v,}{c^{2}+v^{2}-2 c v \cos \phi^{\prime}}, \\
\tan \frac{\phi^{\prime}}{2}=\frac{c-v}{c+v} \tan \frac{\phi}{2}, \\
v(c+v \cos \phi)=v^{\prime}\left(c-v \cos \phi^{\prime}\right), \\
v^{\prime \prime}=\frac{v(c+v \cos \phi+u \sin \phi)}{\sqrt{c^{2}-w^{2}}}=\frac{v^{\prime}\left(c-v \cos \phi^{\prime}+u \sin \phi^{\prime}\right)}{\sqrt{2}}=v^{\prime \prime \prime},
\end{gathered}
$$

where $u$ is the component of $w$ along the axis of $y$.

The last equation indicates that the apparent frequencies $v^{\prime \prime}, \nu^{\prime \prime \prime}$ of the incident and reflected rays, relatively to an observer moving with the same velocity as the mirror, are the samet.

* See the papers by Einstein and Hicks.

$\dagger C f$. Hicks and Einstein, l. $c$. We may show that the expression on the left-hand side represents the apparent frequency of the incident light by applying Lorentz's transfornation so as to pass from a system moving with velocity $w$ to a system at rest. 
It will be noticed that the laws of reflexion depend only on the normal component of the mirror's velocity.

What I wish to point out is that these laws of reflexion can be obtained in a very simple way by supposing that the image of an object is derived by means of the space time transformation:

$$
\begin{aligned}
& x^{\prime}=-\frac{c^{2}+v^{2}}{c^{2}-v^{2}} x+\frac{2 c^{2} v}{c^{2}-v^{2}} t, \quad y^{\prime}=y, \\
& t^{\prime}=\frac{c^{2}+v^{2}}{c^{2}-v^{2}} t-\frac{2 v}{c^{2}-v^{2}} x, \quad z^{\prime}=z .
\end{aligned}
$$

This transformation reduces to the one considered by Voigt *, Lorentz $\dagger$, and Einstein $\ddagger$, if we change the sign of $x^{\prime}$ and put

Since

$$
\mathrm{U}=\frac{2 c^{2} v}{c^{2}+v^{2}}
$$

$$
x^{\prime}=x-\frac{2 c^{2}}{c^{2}-v^{2}}(x-v t), \quad t^{\prime}=t-\frac{2 v}{c^{2}-v^{2}}(a-v t),
$$

it is clear that at any time every point of the moving mirror $(x=v t)$ is transformed into itself. The image of a point moving with the same velocity as the mirror is another point moving with the same velocity, for if dots denote differentiations with regard to $t$

$$
\begin{gathered}
\dot{x}^{\prime}=\dot{x}-\frac{2 c^{2}}{c^{2}-v^{2}}(\dot{x}-v)=\dot{x}, \\
\dot{y}^{\prime}=\dot{y}, \quad z^{\prime}=\varepsilon, \\
\dot{t}^{\prime}=1-\frac{2 v}{c^{2}-v^{2}}(\dot{x}-v)=1 . \\
\therefore \frac{d x^{\prime}}{d t^{\prime}}=\dot{x}, \quad \frac{d y^{\prime}}{d t^{\prime}}=\dot{y}, \quad \frac{d z^{\prime}}{d t^{\prime}} \dot{z}=\dot{z} .
\end{gathered}
$$

The image of a stationary point, on the other hand, is a point moving with the uniform velocity

$$
\mathrm{U}=\frac{2 c^{2} v}{c^{2}+v^{2}}
$$

* Göttinger Nachrichten, 1887, p. 41.

$\dagger$ Amsterdem Proceedings, 1904.

$\downarrow$ Ann. d. Phys. xvii. (1905). 
Further, since

$$
x=-\frac{c^{2}+v^{2}}{c^{2}-v^{2}} x^{\prime}-\frac{2 c^{2} v}{c^{2}-v^{2}} t^{\prime},
$$

we have for a given value of $t^{\prime}$

$$
\begin{aligned}
& x_{1}-x_{2}=-\frac{c^{2}+v^{2}}{c^{2}-v^{2}}\left(x_{1}{ }^{\prime}-x_{2}{ }^{\prime}\right), \\
& y_{1}-y_{2}=y_{1}^{\prime}-y_{2}^{\prime}, \\
& z_{1}-z_{2}=z_{1}{ }^{\prime}-z_{2}{ }^{\prime} .
\end{aligned}
$$

The image of a stationary object, therefore, suffers a contraction proportional to

$$
\frac{c^{2}-v^{2}}{c^{2}+v^{2}}=\sqrt{1-\frac{U^{2}}{c^{2}}}
$$

times its length in a direction parallel to the axis of $x$. This is the proper contraction for velocity $U$ according to the hypothesis put forward by FitzGerald* and Lorentz $\dagger$ to explain the negative result of the famous experiment made by Michelson and Morley.

To show that this transformation gives the correct laws of reflexion of a plane wave we introduce dashed letters into the argument of the periodic function of the reflected wave and make the necessary substitutions. The argument

$$
v^{\prime}\left[t^{\prime}-\frac{x^{\prime} \cos \phi^{\prime}-y^{\prime} \sin \phi^{\prime}}{c}\right]
$$

is then transformed into

$$
\nu^{\prime}\left[\frac{c^{2}+v^{2}-2 c v \cos \phi^{\prime}}{c^{2}-v^{2}} t+\frac{\left(c^{2}+v^{2}\right) \cos \phi^{\prime}-2 c v}{c^{2}-v^{2}} \frac{x}{c}+\frac{y}{c} \sin \phi^{\prime}\right],
$$

and if we compare this with

$$
v\left[t+\frac{x \cos \phi+y \sin \phi}{c}\right]
$$

we obtain the relations (I).

The transformation can evidently be applied to waves of any form.

The formula connecting the components of the electric

* Public Lectures in Trinity College, Dublin.

†'Versuch einer Theoris der Elektrischen Körpern' (1895). See also Larmor's ' Ether and Matter.' 
and magnetic force in the incident and reflected waves are obtained by putting *

$$
\begin{array}{r}
{\left[+\mathrm{E}_{x}^{\prime} d y^{\prime} d z^{\prime}+\mathrm{E}_{y}^{\prime} d z^{\prime} d x^{\prime}+\mathrm{E}_{z}^{\prime} d x^{\prime} d y^{\prime}-c \mathrm{H}_{x}{ }^{\prime} d x^{\prime} d t^{\prime}\right.} \\
\left.-c \mathrm{H}_{y}^{\prime} d y^{\prime} d t^{\prime}-c \mathrm{H}_{z}^{\prime} d z^{\prime} d t^{\prime}\right]
\end{array}
$$

$=\mathrm{E}_{x} d y d z+\mathrm{E}_{y} d z d x+\mathrm{E}_{z} d x d y+c \mathrm{H}_{x} d x d t+c \mathrm{H}_{y} d y d t+c \mathrm{H}_{z} d z d t$,

where $\left(\mathrm{E}_{x}, \mathrm{E}_{y}, \mathrm{E}_{z}\right)\left(\mathrm{H}_{x}, \mathrm{H}_{y}, \mathrm{H}_{z}\right)$ are the components of the electric and magnetic force respectively.

Calculating $\mathrm{E}_{x}{ }^{\prime}, \mathrm{H}_{x}{ }^{\prime} \ldots$ by means of the formulæ

$$
\begin{array}{r}
+\mathrm{E}_{x}^{\prime}=\mathrm{E}_{x} \frac{\partial(y, z)}{\partial\left(y^{\prime}, z^{\prime}\right)}+\mathrm{E}_{y} \frac{\partial(z, x)}{\partial\left(y^{\prime}, z^{\prime}\right)}+\mathrm{E}_{z} \frac{\partial(x, y)}{\partial\left(y^{\prime}, z^{\prime}\right)}-c \mathrm{H}_{x} \frac{\partial(x, t)}{\partial\left(y^{\prime}, z^{\prime}\right)} \\
-c \mathrm{H}_{y} \frac{\partial(y, t)}{\partial\left(y^{\prime}, z^{\prime}\right)}-c \mathrm{H}_{z} \frac{\partial(z, t)}{\partial\left(y^{\prime}, z^{\prime}\right)},
\end{array}
$$

we have

$$
\begin{array}{ll}
\mathrm{E}_{x}{ }^{\prime}=+\mathrm{E}_{x}, & \mathrm{H}_{x}{ }^{\prime}=\mathrm{H}_{x} \\
\mathrm{E}_{y}{ }^{\prime}=-\frac{c^{2}+v^{2}}{c^{2}-v^{2}} \mathrm{E}_{y}+\frac{2 c v}{c^{2}-v^{2}} \mathrm{H}_{z}, & \mathrm{H}_{y^{\prime}}=-\frac{c^{2}+v^{2}}{c^{2}-v^{2}} \mathrm{H}_{y}-\frac{2 c v}{c^{2}-v^{2}} \mathrm{E}_{z}, \\
\mathrm{E}_{z}{ }^{\prime}=-\frac{c^{2}+v^{2}}{c^{2}-v^{2}} \mathrm{E}_{z}-\frac{2 c v}{c^{2}-v^{2}} \mathrm{H}_{y}, & \mathrm{H}_{z}{ }^{\prime}=-\frac{c^{2}+v^{2}}{c^{2}-v^{2}} \mathrm{H}_{z}+\frac{2 c v}{c^{2}-v^{2}} \mathrm{E}_{y} .
\end{array}
$$

These give

$$
\begin{aligned}
& \mathrm{E}_{y}{ }^{\prime}+v \mathrm{H}_{z}{ }^{\prime}=-\left(\mathrm{E}_{y}+v \mathrm{H}_{z}\right), \\
& \mathrm{E}_{z}{ }^{\prime}-v \mathrm{H}_{y}{ }^{\prime}=-\left(\mathrm{E}_{z}-v \mathrm{H}_{y}\right), \\
& \mathrm{H}_{y}^{\prime}-v \mathrm{E}_{z}^{\prime}=-\left(\mathrm{H}_{y}-v \mathrm{E}_{z}\right), \\
& \mathrm{H}_{z}{ }^{\prime}+v \mathrm{E}_{y}{ }^{\prime}=-\left(\mathrm{H}_{z}+v \mathrm{E}_{y}\right) .
\end{aligned}
$$

Relative to an observer moving with velocity $v$ the components of the electric and magnetic force are

$$
\begin{array}{lll}
\mathrm{E}_{x}, & \frac{\mathrm{E}_{y}+v \mathrm{H}_{z}}{\sqrt{1-\frac{v^{2}}{c^{2}}}}, & \frac{\mathrm{E}_{z}-v \mathrm{H}_{y}}{\sqrt{1-\frac{v^{2}}{c^{2}}}} \\
\mathrm{H}_{x}, & \frac{\mathrm{H}_{y}-v \mathrm{E}_{z}}{\sqrt{1-\frac{v^{2}}{c^{2}}}}, & \frac{\mathrm{H}_{z}+v \mathrm{E}_{y}}{\sqrt{1-\frac{v^{2}}{c^{2}}}} .
\end{array}
$$

Hence the above equations indicate that the sum of the tangential components of the two vectors in the incident and reflected waves is zero at the surface of the mirror.

* See a paper by the author, Proc. London Math. Soc. 1909. 
Doppler Effect in Positive Rays in Mydrogen.

The formulæ of transformation for the convection currents and volume density of electricity are obtained from the equation *

$$
\begin{array}{r}
\rho^{\prime} w_{x}{ }^{\prime} d y^{\prime} d z^{\prime} d t^{\prime}+\rho^{\prime} w_{y} d z^{\prime} d x^{\prime} d t^{\prime}+\rho^{\prime} w_{z}^{\prime} d x^{\prime} d y^{\prime} d t^{\prime}-\rho^{\prime} d x^{\prime} d y^{\prime} d z^{\prime} \\
=\rho w_{x} d y d z d t+\rho w_{y} d z d x d t+\rho w_{z} d x d y d t-\rho d x d y d z,
\end{array}
$$

where $\left(\rho w_{x}, \rho w_{y}, \rho w_{z}\right)$ are the components of the convection currents, $\rho$ the volume density of electricity. Thus

$$
\rho^{\prime}=-\frac{2 v}{e^{2}-v^{2}} \rho w_{x}-\frac{c^{2}+v^{2}}{e^{2}-v^{2}} \rho .
$$

Since $\left(w_{x}\right)<c$ it appears that $\rho^{\prime}$ and $\rho$ are of opposite signs.

The University, Manchester.

XCVI. The Doppler Effect in Positive Rays in Hydrogen. $B y$ T. Royos, M.Se., 1851 Exhitition Science Scholart.

T ITTLE is known of the cause of luminescence of the 1 canal rays and of the origin of the discrepancy between their actual velocity and that calculated from the potential drop through which they have fallen. Some explanation of these would probably be obtained if the velocity of the positive rays at different parts of the discharge were compared with the distribution of potential, of electric force, and of the rate of change of the latter. Unfortunately there is no satisfactory method of isolating the different portions between the cathode and the negative glow for the observation of the Doppler effect. At one point of the discharge, however, a comparison is possible, namely, at the point where the cathode glow (Goldstein's " erste Kathodenschicht") commences. Since the canal rays mostly start from the negative glow, we can assume that the commencement of the cathode glow corresponds to the minimum Doppler effect when the cathode is viewed from the anode side. That the cathode glow near its commencement shows the Doppler effect was proved by placing the discharge-tube inclined to the axis of the collimator until light from only the first portions of the cathode glow fell on the slit, the portions nearer the cathode being screened off by the cylinder carrying the cathode. The

* Proc. London Math. Soc. (2), 1909.

† Communicated by the Author. 